Technological University Dublin

DÜBLIN

ARROW@TU Dublin

2007-06-01

\title{
An Experimental Investigation of Parallel Multimedia Streams over IEEE 802.11e WLAN Networks using TXOP
}

Nicola Cranley

Technological University Dublin, nicola.cranley@tudublin.ie

Tanmoy Debnath

Technological University Dublin, Tanmoy.Debnath@tudublin.ie

Mark Davis

Technological University Dublin, mark.davis@tudublin.ie

Follow this and additional works at: https://arrow.tudublin.ie/commcon

Part of the Systems and Communications Commons

\section{Recommended Citation}

Cranley, N., Debnath, T. \& Davis, M. (2007) An experimental investigation of parallel multimedia streams over IEEE 802.11e WLAN networks using TXOP. Proceedings of IEEE International Conference on Communications (ICC 2007), Glasgow, Scotland, 24-28 June, 2007.

This Conference Paper is brought to you for free and open access by the Communications Network Research Institute at ARROW@TU Dublin. It has been accepted for inclusion in Conference papers by an authorized administrator of ARROW@TU Dublin. For more information, please contact arrow.admin@tudublin.ie, aisling.coyne@tudublin.ie,gerard.connolly@tudublin.ie.

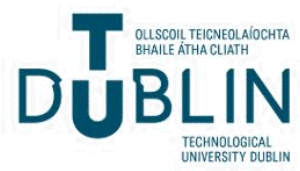




\title{
An Experimental Investigation of Parallel Multimedia Streams over IEEE 802.11e WLAN Networks using TXOP
}

\author{
Nicola Cranley, Tanmoy Debnath, Mark Davis \\ Communications Network Research Institute, \\ School of Electronic and Communications Engineering, \\ Dublin Institute of Technology, \\ FOCAS Institute, \\ Dublin 8, Ireland
}

\begin{abstract}
In this paper we perform an experimental investigation of the IEEE 802.11e TXOP facility to enhance the transmission of parallel multimedia streaming sessions through efficient bandwidth reservation and explicitly consider both the audio and video streams. The delay constraints associated with the audio and video streams that comprise a multimedia session pose the greatest challenge since real-time multimedia is particularly sensitive to delay as the packets require a strict bounded end-to-end delay. We show how the TXOPLimit parameter can be efficiently dimensioned to reduce the transmission delay for the video frames. Due to its frame-based nature, video applications are considered to be bursty as each video frame is typically transmitted as a burst of packets. The size of the burst is related to the size of the video frame and the number of packets required to transmit the video frame. The TXOP facility is particularly suited to efficiently deal with this burstiness since it can be used to reserve bandwidth for the duration of the packet burst. Through experimental investigation, we show that there is a significant performance improvement for the video streams by using the TXOPLimit parameter however there is no such improvement for the audio streams. We show that over-dimensioning the TXOPLimit parameter can cause the video stream to seize too much bandwidth which results in a deterioration in performance for the other competing traffic streams. This deterioration becomes more prominent as the number of parallel multimedia streams increases. We show that there is a performance improvement to all traffic streams by providing differentiated service to the constituent $I, P$, and $B$ video frame types in conjunction with the TXOP facility.
\end{abstract}

Keywords: Multimedia, Video Streaming, Performance Evaluation, Quality of Service, WLAN

\section{INTRODUCTION}

Streaming multimedia over wireless networks is becoming an increasingly important service [1]. This trend includes the deployment of WLANs that enable users to access various services including those that distribute rich media content anywhere, anytime, and from any device e.g. in-home wireless entertainment systems. There are many performance-related issues associated with the delivery of time-sensitive multimedia content using current IEEE 802.11 WLAN standards. Among the most significant are low delivery rates, high error rates, contention between stations for access to the medium, back-off mechanisms, collisions, signal attenuation with distance, signal interference, etc. Multimedia applications, in particular, impose onerous resource requirements on bandwidth constrained WLAN networks. Moreover, it is difficult to provide QoS in WLAN networks as the capacity of the network also varies with the offered load [2].

Providing QoS is difficult since different users, service providers, network administrators, and applications have diverse and sometimes conflicting QoS requirements [3]. For real-time multimedia applications packet loss and packets dropped due to excessive delay are the primary factors affecting the user-perceived quality. Real-time multimedia is particularly sensitive to delay as it has a strict bounded end-toend delay constraint. Every multimedia packet must arrive at the client before its playout time with enough time to decode and display the contents of the packet. For video streams the delay incurred in transmitting the entire video frame from the sender to the client is of particular importance. The loss rates incurred due to packets being delayed past their playout time is heavily dependent on the delay constraint imposed on the video stream. Video streaming applications typically impose an upper limit on the tolerable packet loss. Specifically, the packet loss ratio is required to be kept below a threshold to achieve acceptable visual quality. For example, a large packet loss ratio can result from network congestion causing severe degradation of multimedia quality. Although WLAN networks allow for packet retransmissions in the event of an unsuccessful transmission attempt, the retransmitted packet must arrive before its playout time or within a specified delay constraint. If the packet arrives too late for its playout time, the packet is useless and effectively lost.

In IEEE $802.11 \mathrm{~b}$ WLANs, the AP is a critical component that determines the performance of the network since it carries all of the downlink transmissions to wireless clients and is usually where congestion is most likely to occur. The AP can become saturated due to a heavy downlink load which results in packets being dropped from its transmission buffer and this manifests itself as bursty losses and increased delays [4]. Such losses and delays have a serious impact on multimedia streaming applications. This situation however need no longer apply following the approval of the IEEE 802.11e QoS MAC Enhancement standard which allows for up to four different 
transmit queues with different access priorities [5], allowing the AP to provide differentiated service to different applications and enable to meet their target QoS requirements. The IEEE 802.11e standard also defines a transmission opportunity (TXOP) as the interval of time during which a particular QSTA has the right to initiate transmissions without having to re-contend for access. During an EDCA TXOP, a QSTA is allowed to transmit multiple MPDUs from the same AC with a SIFS time gap between an ACK and the subsequent frame transmission [6]. The duration of the TXOP is determined by the value of the TXOP limit parameter.

This TXOP mechanism is particularly suited to video streaming applications. Video streaming is often described as "bursty" and this can be attributed to the frame-based nature of video. Video frames are transmitted with a particular frame rate. In general, video frames are large, often exceeding the MTU of the network and results in several packets being transmitted in a burst for each video frame where the frequency of these bursts corresponds to the frame rate of the video. The TXOP feature can be used to transmit a burst of video packets corresponding to a single video frame during the allocated TXOP interval.

In this paper we experimentally investigate the performance of providing differentiated service to parallel multimedia streaming applications under heavily loaded conditions using the TXOPLimit parameter. In this work we explicitly consider the performance of both the audio and video streams that comprise the multimedia session. We show that the TXOPLimit parameter does not improve the delivery of the audio samples but significantly improves the delivery of the video frames. We show that by over-dimensioning the TXOPLimit parameter for the video Access Category (AC) causes the performance to deteriorate for the competing traffic in the other ACs and that this deterioration becomes more pronounced as the number of multimedia streams increases. We demonstrate that there is a performance improvement for all ACs by providing differentiated service to the individual constituent I, P, and B video frame types.

The remainder of this paper is structured as follows. Section 2 describes the experimental test bed. In Section 3 the characteristics of the audio and video streams that comprise the multimedia sessions used in the experiments are presented. Section 4 describes how the TXOPLimit parameter is dimensioned for each of the ACs. Section 5 presents the experimental results for the audio, video and background traffic in terms of throughput, delay and loss. Section 6 presents conclusions and directions for future work.

\section{EXPERIMENTAL TEST BED}

To investigate the use of the 802.11e TXOP mechanism for video frame transmission, the video server was set up on the wired network and streamed video to a wireless client via the AP (Figure 1). The AP used was the Cisco Aironet 1200 using the firmware version IOS 12.3(8)JA which allowed us to access the 802.11e/WME capability of the device [7]. The AP was configured with a QoS policy where the Differentiated Services Code Point (DSCP) values in the IP header are used

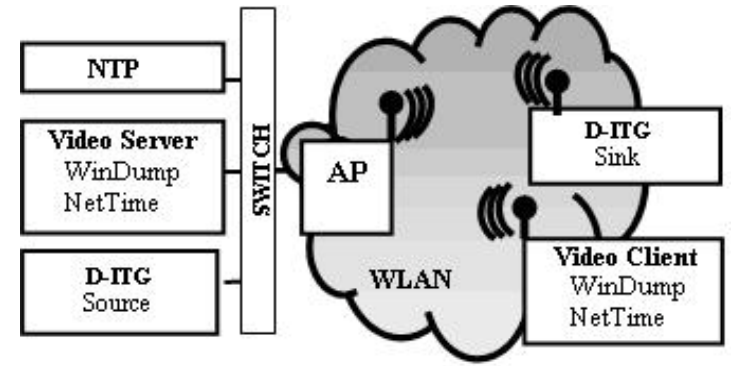

Figure 1 Experimental Test Bed

to apply a particular Class of Service $(\mathrm{CoS})$ to the incoming packets. Each $\mathrm{CoS}$ is then mapped to a particular $\mathrm{AC}$ where the CWmin, CWmax, AIFSN, and TXOP limit parameters can be configured. In the experiments reported here only the TXOP limit parameter is varied and the parameters CWmin, CWmax, and AIFSN were fixed with the original IEEE 802.11 b settings.

The video streaming server consists of a modified version of RTPSender [8]. RTPSender reads from an encoded video file and identifies the different video frame types i.e. I, P, or B frames. The frame type indicator is used to set the IP DSCP value of the packets for this video frame. By modifying the IP DSCP value of video packets for the different frame types the AP can identify the different video frame types and assign them to the appropriate $\mathrm{AC}$ so that they can receive differentiated service as defined by the AP QoS policy.

Both the MultiMedia (MM) client and server used the packet monitoring tool WinDump [9] to log all packets transmitted and received and the clocks of both the client and server are synchronised before each test using NetTime [10]. However, in spite of the initial clock synchronisation, there was a noticeable clock skew observed in the delay measurements and this was subsequently removed using Paxson's algorithm as described in [11]. The delay measured here is the difference between the time at which the packet was received at the link-layer of the client and the time it was transmitted at the link-layer of the sender.

The background traffic was generated using Distributed Internet Traffic Generator (D-ITG) [12]. The background traffic load had an exponentially distributed inter-packet time with a mean offered load of $6 \mathrm{Mbps}$ and an exponentially distributed packet size with a mean packet size of 1024B. The background traffic was transmitted from a wired source station via the AP to a wireless sink station.

\section{Multimedia StREAm Analysis}

In the experiments reported here, the audio and video content was encoded using the commercially available X4Live MPEG-4 encoder from Dicas. In MPEG-4 the audio and video streams are transmitted separately through their own RTP/RTCP port pair. In this paper five different video content clips were used during the experiments. DH is an extract from the film 'Die Hard', DS is an extract from the film 'Don't Say a Word', EL is an extract from the animation film 'The Road to Eldorado', FM is an extract from the film 'Family Man', and finally JR is an extract from the film 'Jurassic Park'. 
TABLE 1: CHARACTERISTICS OF THE VIDEO CONTENT

\begin{tabular}{|c|c|c|c|c|c|c|}
\hline & DH & JR & EL & FM & DS & $\begin{array}{l}\text { Mean Per } \\
\text { Stream }\end{array}$ \\
\hline Frame Rate (fps) & 24.0 & 24.0 & 24.0 & 24.0 & 24.0 & 24.0 \\
\hline Mean Bitrate (kbps) & 1633.0 & 980.0 & 1373.0 & 735.0 & 572.0 & 1058.6 \\
\hline Load I-frames (kbps) & 239.0 & 161.0 & 404.0 & 120.0 & 115.0 & 207.8 \\
\hline Load P-frames (kbps) & 407.0 & 315.0 & 457.0 & 202.0 & 170.0 & 310.2 \\
\hline Load B-frames (kbps) & 987.0 & 504.0 & 512.0 & 413.0 & 287.0 & 540.6 \\
\hline Mean/Max Frame Size (kb) & 35.4 & 27.9 & 40.0 & 35.4 & 39.4 & 35.6 \\
\hline Mean/Max I Frame Size (kb) & $53.7 / 135.4$ & $50.6 / 103.7$ & $109.3 / 214.4$ & $82.0 / 139.9$ & $69.3 / 131.6$ & $73.0 / 214.4$ \\
\hline Mean/Max P Frame Size (kb) & $18.6 / 112.2$ & $17.0 / 89.9$ & $37.1 / 130.1$ & $27.5 / 130.2$ & $23.3 / 116.6$ & $24.7 / 130.2$ \\
\hline Mean/Max B Frame Size (kb) & $6.9 / 200.8$ & $6.4 / 104.3$ & $13.9 / 112.3$ & $10.3 / 83.7$ & $8.7 / 92.4$ & $9.2 / 200.8$ \\
\hline PMR & 35.4 & 27.9 & 40.0 & 35.4 & 39.4 & 35.6 \\
\hline
\end{tabular}

Animated videos are particularly challenging for encoders since they generally consist of line art and as such have greater spatial detail. The video clips were prepared for streaming by creating an associated hint track using MP4Creator from MPEG4IP. The hint track tells the server how to optimally packetise a specific amount of media data. The hint track MTU setting means that the packet size will not exceed in the MTU size.

The audio content was encoded as MPEG-4 Advanced Audio Codec (AAC), $48 \mathrm{kHz}$, and $128 \mathrm{kbps}$ CBR. The audio streams have the following characteristics: mean bit rate $(130.93 \pm 15.27) \mathrm{kbps}$; mean sample size $(341 \pm 40) \mathrm{B}$; maximum sample size 667B; minimum sample size 52B; Peak-to-Mean Ratio (PMR) of 1.96. This video content is approximately 10 minutes in duration and was encoded as MPEG-4 ASP (i.e. I, $\mathrm{P}$, and $\mathrm{B}$ frames) with a frame rate of $24 \mathrm{fps}$, a specified refresh rate of 10 (i.e. an I-frame every 10 frames), GOP sequence (i.e. IPBBPBBPBB resulting in 3 I-Frames, 6 P-frames, and 15 B-frames per second), CIF resolution and a target bitrate of $1 \mathrm{Mbps}$ using 2-pass encoding. In the experiments reported here the hint track MTU is 1024B for all video content types. Although the mean packet size is approximately less than the hint track MTU setting approximately 920B since if the video frame is larger than the hint track MTU setting, several packets are required to send the video frame resulting in a group of packets with a packet size equal to the hint track MTU setting and a smaller packet containing the remainder of the information.

Table 1 shows characteristics of each of the different video streams that were used in the experiments and the average over all content types. It can be seen that high action and animation clips are particularly difficult for the encoder to achieve the target bitrate. Furthermore it can be seen that the combined load of the I and P-frames is less than the load of the B-frames only. This is due to the GOP structure of the video frames since there are on average three I-frames, six Pframes, and fifteen B-frames per second.

\section{Dimensioning THE TXOP Limit PARAMETER}

The distribution of the frame size is used to correctly dimension the TXOP limit parameter as it statistically describes the encoding characteristics of the video stream and the time required to transmit the video frame. The time it takes to transmit a single video packet (Tp) during a TXOP interval is related to the packet size (PSz) and the physical line rate (Rate) which for $802.11 \mathrm{~b}$ has a maximum value of $11 \mathrm{Mbps}$ [13].

$T_{P}=(P S z /$ Rate $)+(2 * S I F S)+$ Ack

$N p$ is the number of packets required to transmit the video frame of size $F S z$ and is given by,

$N_{P}=(F S z / P S z)$

The TXOP limit parameter $T X O P_{N}$ is set to the number of packets required to transmit the video frame $N p$ multiplied by the time it takes to transmit each packet $T p$ during the TXOP interval. The TXOP limit parameter is an integer value in the range $(0,255)$ and gives the duration of the TXOP interval in units of $32 \mu \mathrm{s}$. If the calculated TXOP duration requested is not a factor of $32 \mu \mathrm{s}$, that value is rounded up to the next higher integer that is a factor of $32 \mu \mathrm{s}$. The maximum allowable TXOP limit is $8160 \mu$ s with a default value of $3008 \mu$ s [6].

$\operatorname{TXOP}_{N}=\left\lceil N_{P} * T_{P}\right\rceil$

When there are no more packets to be sent during the TXOP interval and the channel becomes idle again, the 802.11 Hybrid Controller (HC) may sense the channel and reclaim the channel after a duration of PIFS after the TXOP.

\section{A. Test Case Scenarios}

In all cases the $\mathrm{AC}$ queues were configured with IEEE 802.11b settings for CWmin, CWmax, and AIFSN while the value for TXOPLimit parameter is varied. The $802.11 \mathrm{e}$ standard defines four $\mathrm{AC}$ queues into which different traffic streams can be directed: Voice (VO), Video (VI), Best-Effort (BE), and Background (BK). In this work we investigate a number of different test cases. For the purposes of comparison Case $\mathbf{A}$ is used as a reference scenario and where the AP uses the default $802.11 \mathrm{~b}$ settings and all traffic streams are directed through a single queue.

Since we have found that the load from the B-frames is approximately equal to the combined load of the I and $\mathrm{P}$ frames we investigate two key scenarios: where all video frames regardless of frame type are transmitted through the VI $\mathrm{AC}$ and where the I and P frames are transmitted through the 
TABLE 2: TEST CASES AND CDF OF FRAMES TRANSMITTED IN A SINGLE TXOP

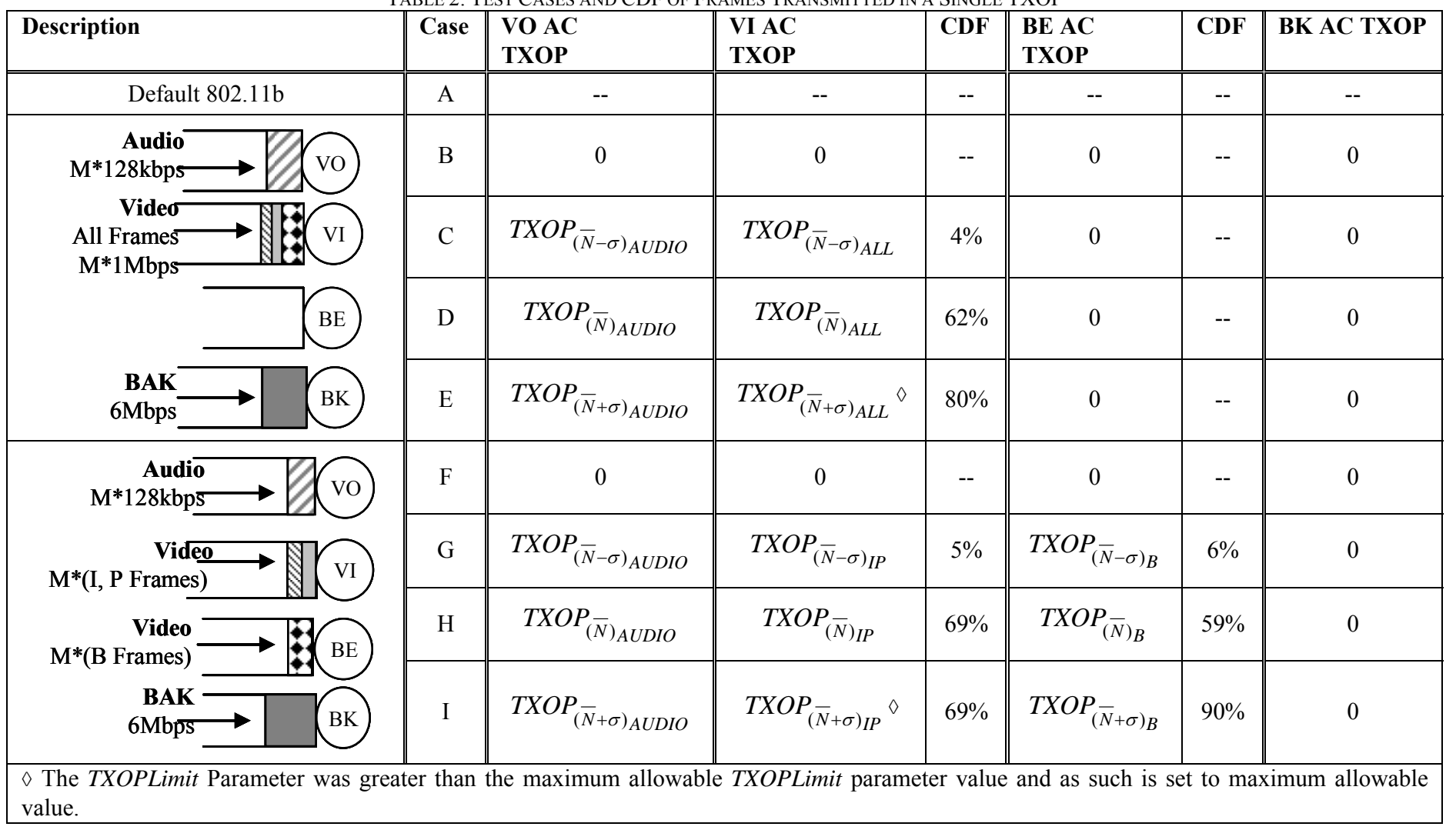

VI AC and the B frames are transmitted through the BE AC.

In Cases $\mathbf{B}$ to $\mathbf{E}$ the audio streams are transmitted through the VO AC queue; the video streams are transmitted through the VI AC queue, and a background traffic load of $6 \mathrm{Mbps}$ is transmitted through the BK AC queue as shown in Table 2. In Case B the TXOPLimit parameter is set to 0 . In Case D the TXOPLimit parameter is related to the mean number of packets $(\bar{N})$ required to transmit an audio sample i.e. $\operatorname{TXOP}_{(\bar{N})_{A U D I O}}$ and all video frames irrespective of frame type i.e. $\operatorname{TXOP}_{(\bar{N})_{A L L}}$. Similarly in Cases C and E the TXOPLimit parameter is related to the mean plus and minus one standard deviation of the number of packets i.e. $(\bar{N}+\sigma)$ and $(\bar{N}-\sigma)$ required to transmit the audio and video frames.

In Cases $\mathbf{F}$ to $\mathbf{I}$ the audio streams are transmitted through the VO AC queue, the I and P video frames are transmitted through the VI AC queue, the B video frames are transmitted through the $\mathrm{BE} \mathrm{AC}$ queue and the background traffic load is transmitted through the BK AC queue as shown in Table 2. In Case $\mathrm{F}$ the TXOPLimit parameter is set to 0 . In Case $\mathrm{H}$ the TXOPLimit parameter is related to the mean number of packets $(\bar{N})$ required to transmit an audio sample i.e. $\operatorname{TXOP}_{(\bar{N})_{A U D I O}}$, I and P video frames i.e. $\operatorname{TXOP}_{(\bar{N})_{I P}}$ and B video frames i.e. $\operatorname{TXOP}_{(\bar{N})_{B}}$. In Cases $\mathrm{G}$ and $\mathrm{I}$ the TXOPLimit parameter is related to the mean plus and minus one standard deviation of the number of packets i.e. $(\bar{N}+\sigma)$ and $(\bar{N}-\sigma)$ required to transmit the audio, $\mathrm{I}$ and $\mathrm{P}$ video frames and $B$ video frames.

These test cases have been summarized in Table 2. For example, in Case D all video frames are transmitted through the VI AC queue. From the CDF of the video frame sizes $62 \%$ of video frames can be transmitted in a single TXOP using the mean number of packets to dimension the TXOPLimit parameter whereas in Case E $80 \%$ of video frames can be transmitted in a single TXOP when the mean plus one standard deviation is used to dimension the TXOPLimit parameter. Since all audio samples can be transmitted in a single TXOP, it is expected that the TXOPLimit parameter of the VO AC queue will not have a significant effect on the endto-end delivery of the audio samples.

\section{RESUlts}

For video streaming applications, not only is the end-to-end packet delay important, but also the delay incurred when transmitting the entire video frame from the sender to the client. Video streaming is often described as "bursty" and this can be attributed to the frame based nature of video. Video frames are transmitted with a particular frame rate and are generally large, often exceeding the MTU of the network which results in a number of packets being transmitted in a burst for each video frame. A video frame cannot be decoded or played out at the client until all or most of the constituent video packets for the frame are received correctly and on time. For this reason, we consider the delay required to transmit the entire video frame. 


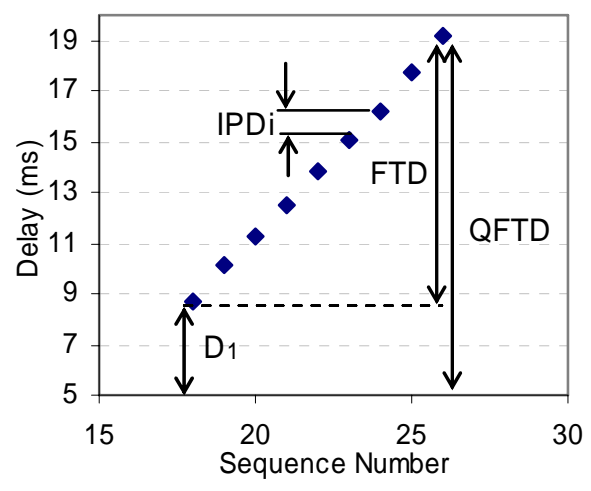

Figure 2 Relationship between IPD, FTD, and QFTD for a single video frame

In a WLAN environment, the bursty behaviour of video traffic has been shown to result in a sawtooth-like delay characteristic [14]. To describe this sawtooth characteristic we have defined the Inter-Packet Delay (IPD) as the difference in the measured delay between consecutive packets within a burst for a video frame at the receiver. In our analysis, we focus on the video Frame Transmission Delay (FTD), i.e. the end-to-end delay incurred in transmitting the entire video frame. The video frame delay is related to the number of packets required to transmit the entire video frame. The FTD is measured as the sum of the IPD for each packet required to transmit the entire video frame where the frame consists of $N$ packets. The QFTD is the FTD plus the transmission delay (D) for the first packet of the video frame to reach the client. Figure 2 shows the relationship between the IPD, FTD, and QFTD for a single video frame.

$$
\begin{aligned}
& F T D=\sum_{i=2}^{N} I P D_{i} \\
& Q F T D=D_{1}+F T D
\end{aligned}
$$

In the experiments the number of multimedia streams was increased from 2 to 5 parallel streams for each of the different test cases in addition to a fixed offered mean background traffic load of $6 \mathrm{Mbps}$ transmitted through the BK AC. For the audio streams the mean packet delay is the measured quantity of interest since a complete audio sample can be contained within a single packet. For the video stream the QFTD is measured, since in general a number of packets are required to transmit a single video frame.

Figure 3 shows the mean delay and loss measures with increasing values for the TXOPLimit parameter for Cases B to $\mathrm{E}$ with an offered background traffic load of 6Mbps. Figure 3(a) and (c) show the mean QFTD and packet delay for the video and audio streams respectively as the number of parallel streams is increased while Figures 3(b) and (d) show the loss rates for the audio and video streams.

It can be seen in Figures 3(a) and (b) that as the TXOPLimit parameter is increased for the video streams, the QFTD is reduced. The system can support 3 parallel video streams that satisfy a tolerable loss rate constraint of 5\%. Case D exhibits the best performance having a QFTD of $18 \mathrm{~ms}$ and loss rate of $3 \%$ for 3 parallel multimedia streams. Increasing the TXOPLimit parameter to the mean plus one standard deviation as in Case E increases the QFTD. In contrast, it can be seen that the TXOPLimit parameter does not improve the end-toend delay incurred transmitting audio samples. This is to be expected since an audio sample can be contained within a single packet and as such the VO AC only needs to win a single transmission opportunity to transmit a complete audio sample. However by comparing the performance of the audio and video streams it can be clearly seen that as the TXOPLimit parameter of the VI AC is increased, the performance (in terms of delay and loss) of the competing audio streams in the VO AC deteriorates.. This is particularly evident as the number of parallel multimedia streams is increased. This is due to the fact that usage of the TXOP is not wasteful since when the AC queue has won a TXOP and has no more packets to send during the TXOP interval, the HC senses the channel as idle and reclaims the channel after a duration of PIFS after the TXOP. As the number of video streams is increased the buffer occupancy of the VI AC queue is also increased which in turn increases the likelihood that the VI AC queue will make use of the full duration of the TXOP interval to transmit the enqueued video packets. Furthermore, as the TXOPLimit parameter of the VI AC is increased it contends for access to the medium more often and as such gains access to the medium for longer intervals each time it wins a transmission opportunity. This in turn increases the waiting time for the VO $\mathrm{AC}$ before it can contend for access to the medium thereby increasing the end-to-end delay for the audio samples.

Figure 4 shows the mean delay and loss measures of the audio and video streams with increasing values for the TXOPLimit parameter for Cases $\mathrm{F}$ to $\mathrm{I}$ with an offered background traffic load of $6 \mathrm{Mbps}$. In this scenario the I and $\mathrm{P}$ frames of the video stream are transmitted through the VI AC while the $\mathrm{B}$ frames are transmitted through the $\mathrm{BE} \mathrm{AC}$ queue. The results show that only 3 multimedia streams can be supported satisfying delay and loss constraints. It can be seen that as the TXOPLimit parameter is increased for the VI and $\mathrm{BE} \mathrm{AC}$ queues both the QFTD and loss rate are significantly reduced.

By comparing Figures 3 and 4 it can be seen that the performance of both the audio and video streams in terms of both the loss rate and delay is improved by transmitting the I and $\mathrm{P}$ frames of the video stream through the VI AC and the B frames through the BE queue as in Cases F-I. Figure 3 (e) and 4 (e) show the percentage throughput of the background traffic for Cases B-E and Cases F-I. These figures show that the performance trade-off between the different AC using the TXOP facility becomes more pronounced in Cases B-E and that the throughput of the background traffic is greater in Cases F-I. 


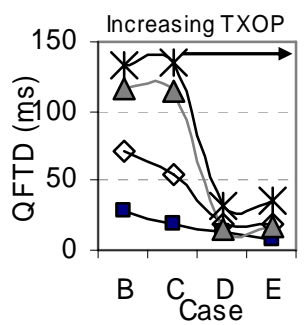

(a) Video

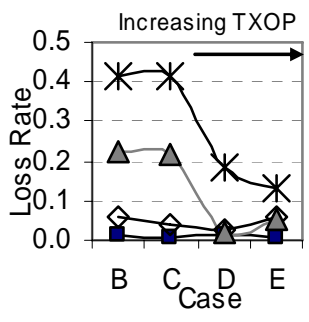

(b) Video

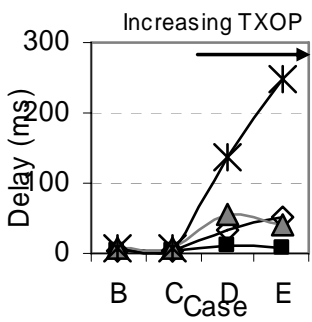

(c) Audio

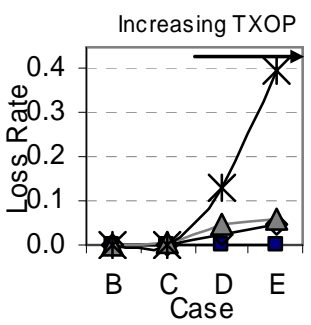

(d) Audio

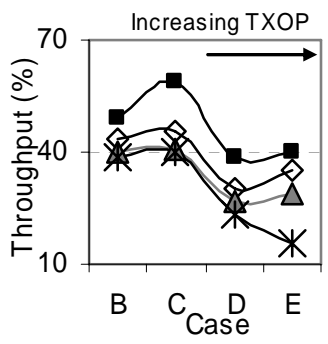

(e) Background

Figure 3 Cases B-E (a) Video QFTD

(c) Video Stream Loss Rate

(b) Audio Sample Delay

(d) Audio Stream Loss Rate (e) Background Traffic Percentage Throughput

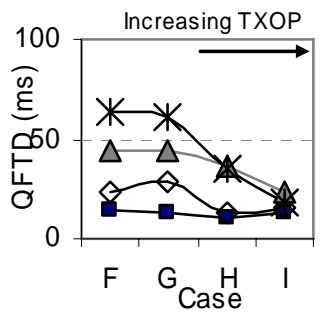

(a) Video

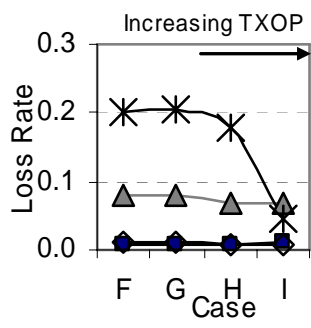

(b) Video

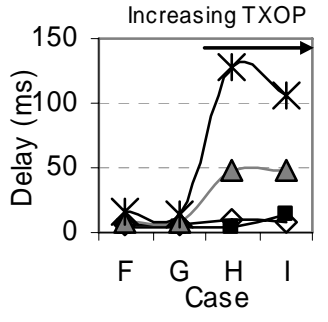

(c) Audio

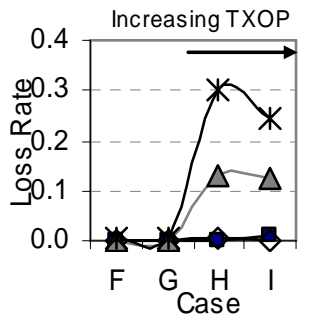

(d) Audio

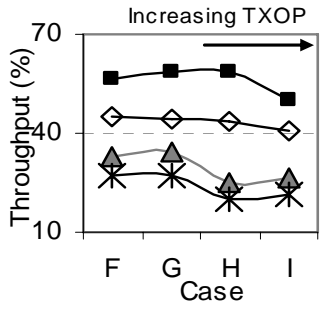

(e) Background

Figure 4 Cases F-I (a) Video QFTD

(e) Background Traffic Percentage Throughput

(c) Video Stream Loss Rate

(b) Audio Sample Delay

(d) Audio Stream Loss Rate

\section{$\curvearrowright-3 \mathrm{AVStreams}$}

$\triangle 4$ AVStreams
By contrast in Case A the AP is configured with the default $802.11 \mathrm{~b}$ settings. When there is no background traffic the QFTD for the video streams increases from $9 \mathrm{~ms}$ with 2 parallel video streams to $26 \mathrm{~ms}$ with a loss rate of $5 \%$ for 5 parallel multimedia streams. When $6 \mathrm{Mbps}$ of background traffic is introduced, the AP becomes saturated resulting in buffer overflow. The throughput of the background traffic load is reduced to $73 \%$ while the video stream suffers a mean QFTD of 91ms and loss rate of 59\% which is unacceptable for multimedia streaming applications. Providing differentiated service to the different traffic streams in conjunction with the TXOP facility provides a significant performance improvement over the default 802.11 b configuration.

\section{CONCLUSIONS}

In this paper, we have experimentally investigated the use of the TXOP facility for streaming parallel multimedia sessions over IEEE 802.11e WLAN networks under heavily loaded conditions. Multimedia streams consist of an audio and video stream. Video is a frame-based media where video frames are transmitted from the server to the client at regular intervals that is related to the frame rate of the video. In general, several packets are required to transmit a single video frame. The video frame cannot be decoded at the client until all the packets for the video frame have been received. In contrast, audio samples are transmitted at regular intervals and each audio sample can be contained within a single packet. In this paper we exploit the periodic packet bursts that characterize video streaming applications to reduce the transmission delay for video frames through tuning of the TXOPLimit parameter.

In this paper we have experimentally shown that when using the $802.11 \mathrm{~b}$ settings 5 multimedia sessions can be supported when there is no background traffic. However, when $6 \mathrm{Mbps}$ of background traffic is introduced no multimedia sessions can be supported and the background traffic experiences a $27 \%$ drop in throughput. In contrast when using $802.11 \mathrm{e}$ three multimedia sessions can be supported satisfying delay and loss rate constraints in the presence of $6 \mathrm{Mbps}$ of background traffic using the TXOP facility. The results show that the TXOPLimit parameter does not improve end-to-end delay for audio samples but significantly improves the end-to-end delay incurred transmitting a single video frame. By over-dimensioning the TXOPLimit parameter for the video streams, the audio streams suffer a performance deterioration since the VI AC queue is effectively taking bandwidth from the other ACs. This performance deterioration becomes more pronounced as the number of parallel multimedia streaming sessions is increased. By providing a differentiated service to the constituent video frame types through transmitting the $\mathrm{I}$ and $\mathrm{P}$ frames through the VI AC queue and the $\mathrm{B}$-frames through the $\mathrm{BE} \mathrm{AC}$ queue, there is a performance improvement in terms of reducing the performance imbalance between the audio and video streams in terms of reduced loss rates and delay. Furthermore by providing prioritized access to the different frame types we can reduce the likelihood of packets relating to I or $\mathrm{P}$ frames being lost since these frames have a higher priority and a greater impact on the end-user QoS over B frames. Further research is being conducted to provide prioritized access to the 
audio streams and video streams and increase the number of parallel multimedia sessions that can be supported through an appropriate tuning of the AIFSN, CWmin, CWmax settings in conjunction with the TXOPLimit parameter.

\section{ACKNOWLEDGEMENT}

The support of the Science Foundation Ireland, grant 03/IN3/1396, under the National Development Plan is gratefully acknowledged.

\section{REFERENCES}

[1] Insight Research Corp., "Streaming Media, IP TV, and Broadband Transport: Telecommunications Carriers and Entertainment Services 2006-2011", Insight Research Corp., April 2006, [Online]. Available: http://www.insightcorp.com/reports/IPTV06.asp

[2] N. Cranley, M. Davis, "Performance Analysis of Network-level QoS with Encoding Configurations for Unicast Video Streaming over IEEE 802.11 WLAN Networks", WirelessCom 2005, Maui, Hawaii, June 2005

[3] Q. Zhang, W. Zhu, Y-Q. Zhang, "End-to-End QoS for Video Delivery Over Wireless Internet", in Proc. of the IEEE, Vol. 93, No. 1, January 2005

[4] N. Cranley, M. Davis, "The Effects of Background Traffic on the End-to-End Delay for Video Streaming Applications over IEEE 802.11b WLAN Networks", IEEE Personal Indoor Mobile Radio Communications (PIMRC) 2006, Helsinki, Finland, September 2006

[5] Q. Ni, "Performance Analysis and Enhancements for IEEE 802.11e Wireless Networks", IEEE Network, Vol. 19, No. 4, July/August 2005, pp. 21-27.

[6] IEEE Std 802.11e, 2005 Edition, IEEE Standards for Local and Metropolitan Area Networks: Specific requirements Part 11: Wireless LAN Medium Access Control (MAC) and Physical Layer (PHY) specifications Amendment 8: Medium Access Control (MAC) Quality of Service Enhancements

[7] Cisco Aironet 1200, http://www.cisco.com/en/US/products/hw/wireless/ps430/index.ht $\mathrm{ml}$

[8] RTPTools, http://www.cs.columbia.edu/IRT/software/rtptools/

[9] WinDump, http://windump.polito.it/

[10] NetTime, http://nettime.sourceforge.net/

[11] S. B. Moon, P. Skelly, D. Towsley, "Estimation and Removal of Clock Skew from Network Delay Measurements", in Proc. of IEEE InfoComm'99, March 1999

[12] Distributed Internet Traffic Generator (D-ITG), http://www.grid.unina.it/software/ITG/download.php

[13] B-S Kim, S-W Kim, Y. Fang, T.F. Wong, "Two Step Multipolling MAC Protocol for Wireless LANs", IEEE JSAC in Communications, Vol. 23, No. 6, June 2005

[14] N. Cranley, M. Davis, "Delay Analysis of Unicast Video Streaming over WLAN", 2nd IEEE International Conference on Wireless and Mobile Computing, Networking and Communications, WiMob 2006, Montreal, Canada, June 2006 